\title{
Snit theory: effects of supernova G11.2-0.3- current events SN 1054 \& 1006 and cholera environment entry.
}

\author{
Sokeland WP* \\ Retired Heat Transfer Expert, Spacecraft and Turbine Engines, Oakland City, Indiana 47660, USA
}

\begin{abstract}
The Supernova and Nova Impact Theory, SNIT, proposes correlation between the right ascension locations of supernova remnants and a 180 degree longitude segment of the earth's sphere where the major effects of debris stream impact occur at termini. One region of time presented will represent the debris stream impact of supernova G11.2-0.3 and will be assumed to have caused the Dalton sunspot minimum from 1790 to 1830 due to the scattering of sunlight by small incoming supernova debris particles. The discovery of the Quadrantids meteor shower during the time period of study suggests supernova G11.2-0.3 is the mechanism that produced the Quadrantids. Recent events caused by acting supernovas 1006 and 1054 are included in this work.
\end{abstract}

Keywords: Warming, sunspots, supernova, flu, volcano, ice, Antarctica, cholera.

Accepted on March 10, 2018

\section{Introduction}

The previous works by the author noted many supernova and nova debris streams impacting our planet and showed possible correlation of numerous effects generally at the beginning of the debris streams [1-4]. This work will concentrate on the effects of the most recent, past, supernova debris stream, G11.2-0.3, and include occurrences of recent effects of SN 1006, SN 1054, and nova WZ Sagittae.

The age of SN 11.2-0.3 is still stated as $1900 \pm 500$ years, but recent research has changed the range of the distance as 14,000 to 23,000 light years [5]. If the 14.000 ly distance is used in conjunction with a base year of 2010 AD, and the beginning of the Dalton sunspot minimum of 1790 AD will be an ETA of 220 years ago, ya. The date the visible light from the supernova occurred or age assuming $88.2325 \%$ light speed for the debris would be 2,087 years ago which resonably coincides with the central value of the stated age. The ranges of values for distance and age for the SN 11.2-0.3 remnant allows a degree of variation, and the numbers in the previous calculation are used for the example of interest.

\section{SN 11.2-0.3 Past Events and Current Events}

\section{SN 11.2-0.3 time period relocation and antarctic heating data}

SN 11.2-0.3 shown as the red arrow of Figure 1 is being relocated due to the new age data gives an ETA of 220 ya which agrees with the Commander Islands Extinction of 266 [1]. This move of the ETA location of SN 11.2-0.3 causes two unknown supernovas to exist in Figure 1 shown as black arrows. Evidence for moving the ETA of SN 11.2-0.3 is the discovery of the Quadrantids meteor shower of 1820 [6]. The right ascension of the radiant for the Quadrantids is $15^{\mathrm{h}} 28^{\mathrm{m}}$ and in reasonable agreement with the right ascension of SN 11.2-0.3 of ${ }^{18 \mathrm{~h}} 11.5 \mathrm{~m}$ [5]. Since the debris particles are positively charged and have pasted through earth's magnetic field, the glowing lines of the meteors being magnetically deflected will not point directly to the source of the debris stream.

The most important data when trying to match the stream of a meteor shower with a supernova explosion will be the CAM date of a terminus which will indicate a yearly repetition of maximum meteor density. In this case the eastern terminus CAM date is December 22 and the meteor shower occurs yearly from December 28 to January 12.

The ice melt shown in Figure 1 agrees with the timing of the Dalton sunspot minimum and the timing agrees with the probable energy input of SN G11.2-0.3.

\section{Large volcanic event and year without a summer event}

Mount Tambora eruption was in 1815 near the time of maximum ice melt shown in Figure 1 indicating the probability of maximum debris particle density impacting the earth magnetic field and providing the maximum energy pulse into the earth's core that produces the largest historical volcanic eruption [9]. The Tambora eruption has been credited as the trigger to the Year without a Summer. It is possible that both effects were due to the incoming SN 11.2-0.3 debris stream.

The termini locations for SN 11.2-0.3 are western terminus 35E and the eastern terminus $145 \mathrm{~W}$. The remnant's declination is in the southern hemisphere at 19 degrees providing the location for maximum impact by the western terminus to displace arctic air mass into the western hemisphere during the summer months of 1816 [10].

Both of these events were discussed in section 13 Polar Air Mass Displacement [2]. It should also be noted that both these events occur near the time of maximum ice melt shown in Figure 1 for the time period of discussion which would agree with the year of impact of maximum particle density for the time period of existence of the particle debris stream of the supernova.

The volcanic eruption of Mount Mayon on January 25, 2018 in 


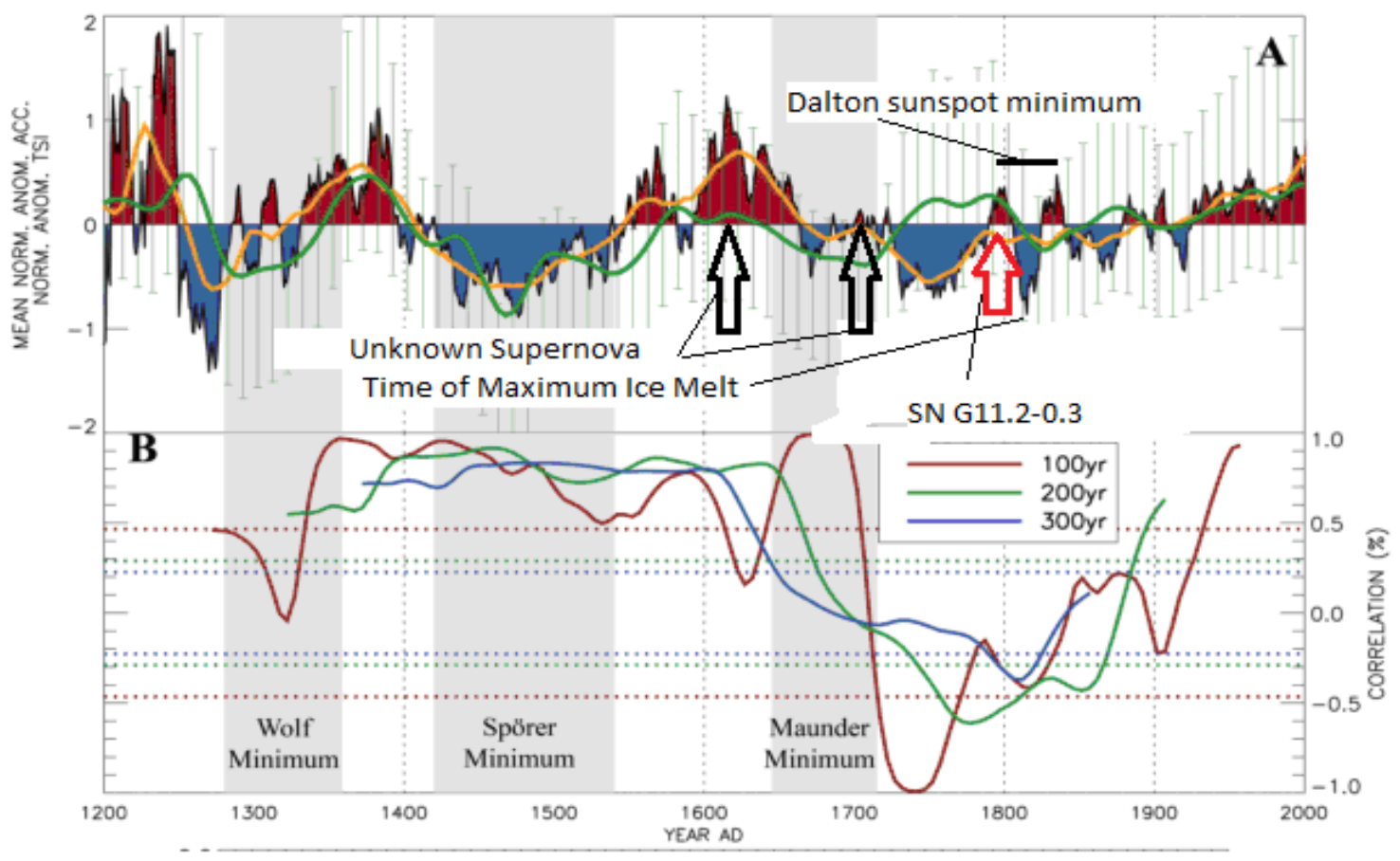

Figure 1. Antarctica Ice Melt and Dalton Sunspot Minimum [7,8].

the Philippines is an event that is associated with the SN 1006 debris stream of today. The SNIT theory proposes there are larger eruptions to come as the maximum density point in time is approached for SN 1006. The phenomena of the Year without a Summer may also repeat.

\section{Drought and Fox-bats Dying}

In Australia the drought of 1789 to 1791 was severe enough to dry up the grass in 1790 and in 1791 Fox-bats fell from the air as they died [11]. With a deflection for the western terminus, 35E, of SN 11.2-0.3 similar to the deflection for today's SN 1006 for Siaga antelope kill of 65 longitudinal degrees, Australia is favorably located for incoming high particle density from the western terminus debris stream [2]. The Australian drought is similar to the droughts of east Africa for the eastern terminus of SN 1054 in 2005, 2006 and the western terminus of SN 1006 in 2011. The 2011 drought in east Africa was the worst drought in the region for 60 years. It should be noted that droughts and animal dying in groups are located in time at the beginning of the debris stream impact.

\section{New Madrid Earthquake}

The New Madrid earthquake of 1811 was an unusual earthquake because it is remote from a plate boundary. It is located near the eastern terminus of SN 11.2-0.3 in the Midwestern USA [12].

\section{Diseases}

The plague in Egypt 1801, 1812; Istanbul 1812; Malta and Romania 1812 all appear to be associated with the western terminus of SN 11.2-0.3 at 35E longitude. The time location of the disease infestation is 11 years after impact of the debris stream. The similar event for today is the pandemic of swine flu in China and the USA is occurring 11 years after the impact of SN 1054 which began in 2007. The first Cholera pandemic was in Asia and Europe from 1816 to 1826 [13]

A minor outbreak of the swine flu from SN 1054 was purposed due to the travel of the debris stream from east to west in 200910 in section 6, Debris stream's Swine Flu [2].

The environmental entry of the cholera germ from the SNs 1006 and 1054 debris streams is proven by the termini longitudinal locations matching the cholera outbreak locations on our planet and the dates of impact for the SNs also matching the beginning of the large cholera outbreaks. The cholera germ infects open water supply in the area of impact for the incoming debris streams.

The first cholera pandemic 1816-1826 killed 100,000 people and the second cholera pandemic occurred from 1829 to 1851 [13]. This pandemics begin during the time period of study of SN 11.2-0.3 effects and the inference that the cholera germ origin is the supernova's debris stream occurs. The time range is so large that the debris stream from nova WZ Sagittae may also be involved. When the cholera pandemic events are considered as results of impacts of exploding star debris streams, data concerning recent large cholera outbreaks provide the subSaharan Africa outbreak beginning in 2007 and the Sierra Leonean, west Africa outbreak beginning in 2012 [14]. The outbreak in sub-Saharan Africa had reached 128,548 cases and 4,053 deaths by February 12, 2009 [14]. The beginning dates of these large outbreaks coincide with the initial impact times of SNs 1054 and 1006, respectively. Figure 2 termini locations show the location for SN 1006's western terminus is correct for the 2012 outbreak and the eastern terminus location for SN 1054 is correct for the 2007 outbreak. The longitudinal location for the eastern terminus, a high debris density point, of SN 1054 shown in Figure 2 is longitudinally central to the sub-Saharan population providing an explanation for the magnitude of the cholera outbreak that began in 2007. 
The locations of both large African cholera outbreaks are shown in Figure 3(a). The infected area of a termini outbreak is probably circular in shape and a high percentage of this area of the Sierra Leonean outbreak could be in the Atlantic Ocean. Confined impact areas to oceans explain large marine animal kills that appear on beaches throughout the world. The large infected area for the sub-Saharan outbreak generally covers land and as a result many humans die.

\section{Consecutive SN 1054 Events}

It is of interest to note that the Thomas wild fire of southern California 2017-18, the largest wild fire ever in the USA, agrees with the CAM date of December 12 as the beginning date of the California fire was December 4 . The deflected western terminus of SN 1054 caused the out of season Santa Ana winds that spread the wild fire. In addition since the SN 1054 is now crossing the USA, we are experiencing a large flu outbreak. The events are consecutive in a west to east direction that is consistent with the particle stream focal point as shown in Figure 2. The USA flu outbreak has decreased in the west and this is consistant with the focaling point passing over the western states first.

\section{South Korean Olympic's Strong Winds 2018}

The mid-February strong winds that postponed South Korean Olympic events match with the particle stream focal point of SN 1006 traveling from east to west from the CAM date of the eastern terminus of November 2 when using the rule of one longitude degree per day as the focal points velocity and

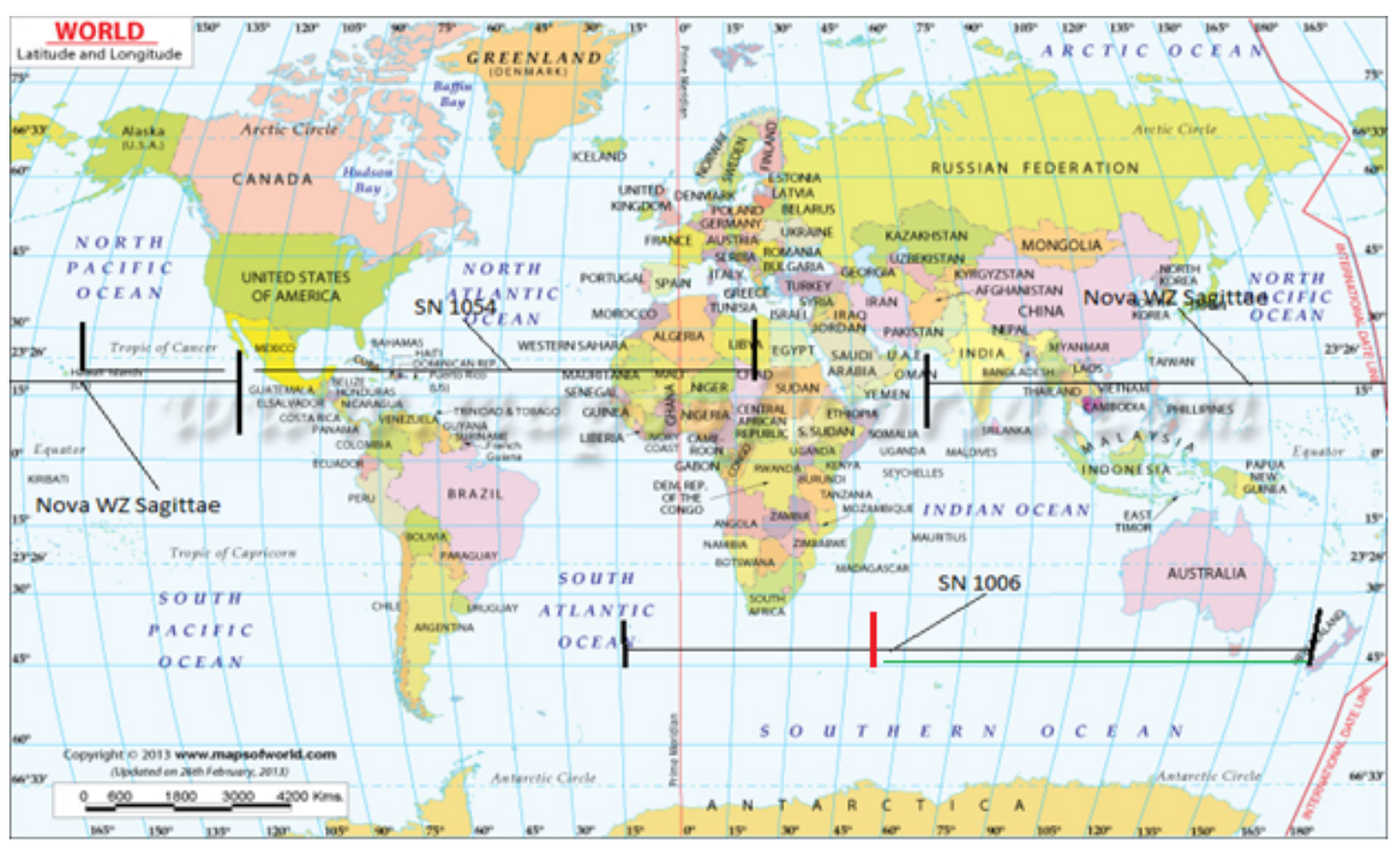

Figure 2. Sectors of Active Supernova and Nova Particle Streams [15].

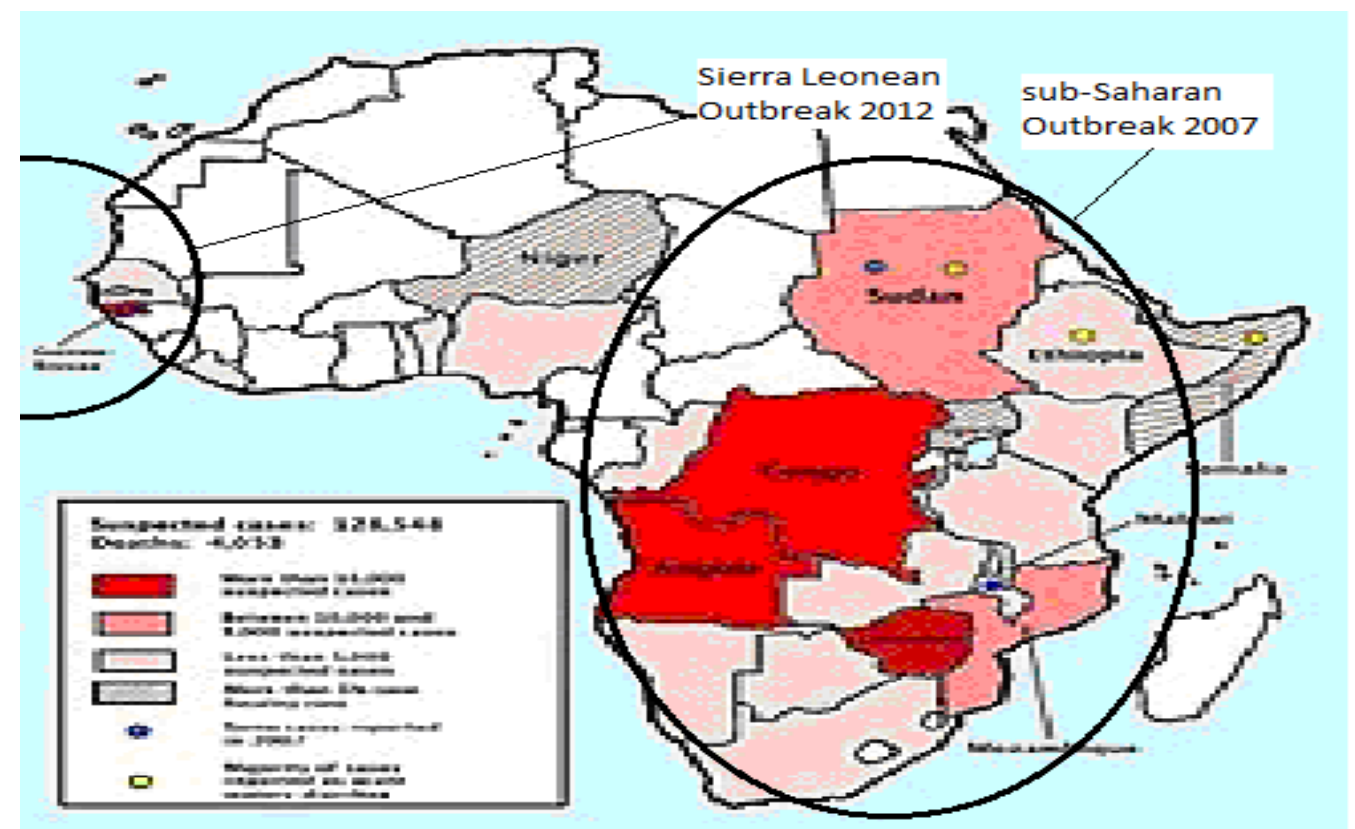

Figure 3(a). Recent African Cholera Outbreaks [14]. 
considering a 30 day width for the northern western terminus focal point Figure 2 [1].

\section{Antarctic Sea Ice Melt 2016-17}

The reported figure in reference 10 is shown as Figure 3(b)

The blue areas that are touched by the thick black, red, and green lines denote the sea ice melts in Antarctica presented in early December 2016. The noted melt areas occurred in November. The thinning of the melted areas for the black and red lines caused by the SN 1006 debris stream occurred in May; however, the exposure did not occur until the December presentation of the data by NASA. If the controlling factors of the melt areas did not change, similar melt areas should be expected in early December 2017.

Figure 3(c) showd the hot spots delivered by the debris stream of SN 1006 in May 2017 that correlate with Antarctic melting in May of the same year.
The southern circled hotspots in Figure 3(c) correspond with the sea ice melt locations in Antarctica in November 2017 due to May 2017 thinning and the northeastern hotspot corresponds to the maximum temperature in Pakistan. The temperature data is presented as a monthly average by Weather Bell. The implied motion of the deflected hotspot indicates India will not be threatened by the May northwestern hotspot of SN 1006 for a number of years.

Figure 4 is the same data as Figure 3(b) and is shown for comparison purposes.

Comparing Figure 4 and Figure 5 it should be noted that the melt for the western terminus, black line, is stronger in 2017 and the deflected melt area, red line, appears to be weaker in 2017. The red arrow in Figure 5 indicates the shift in the deflection of the SN 1006 debris stream due to the change in the sun's magnetic field. The shift is to the west and this should be the same direction for the shift of the northern tine's hotspot from

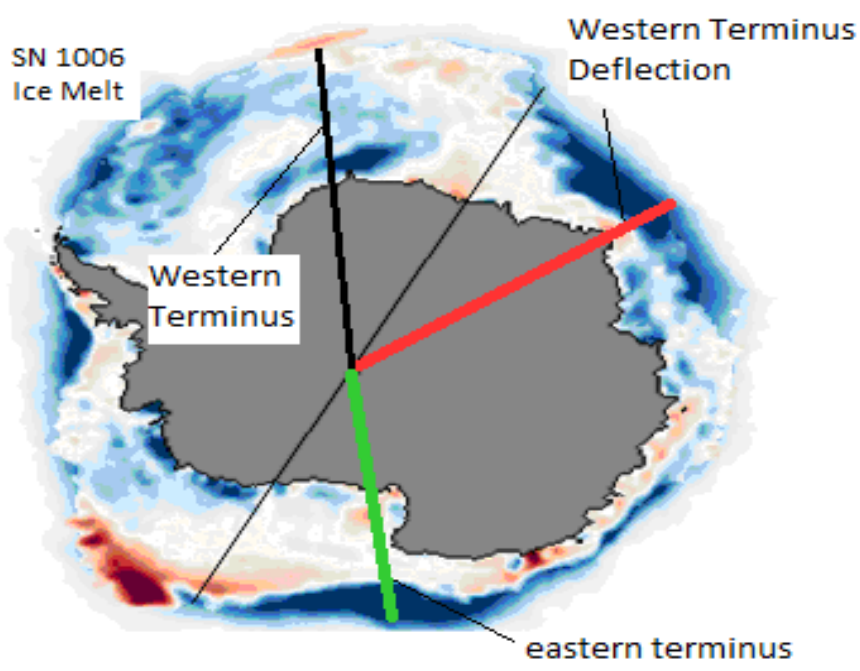

Figure 3(b). December 42016 New Melt Locations.

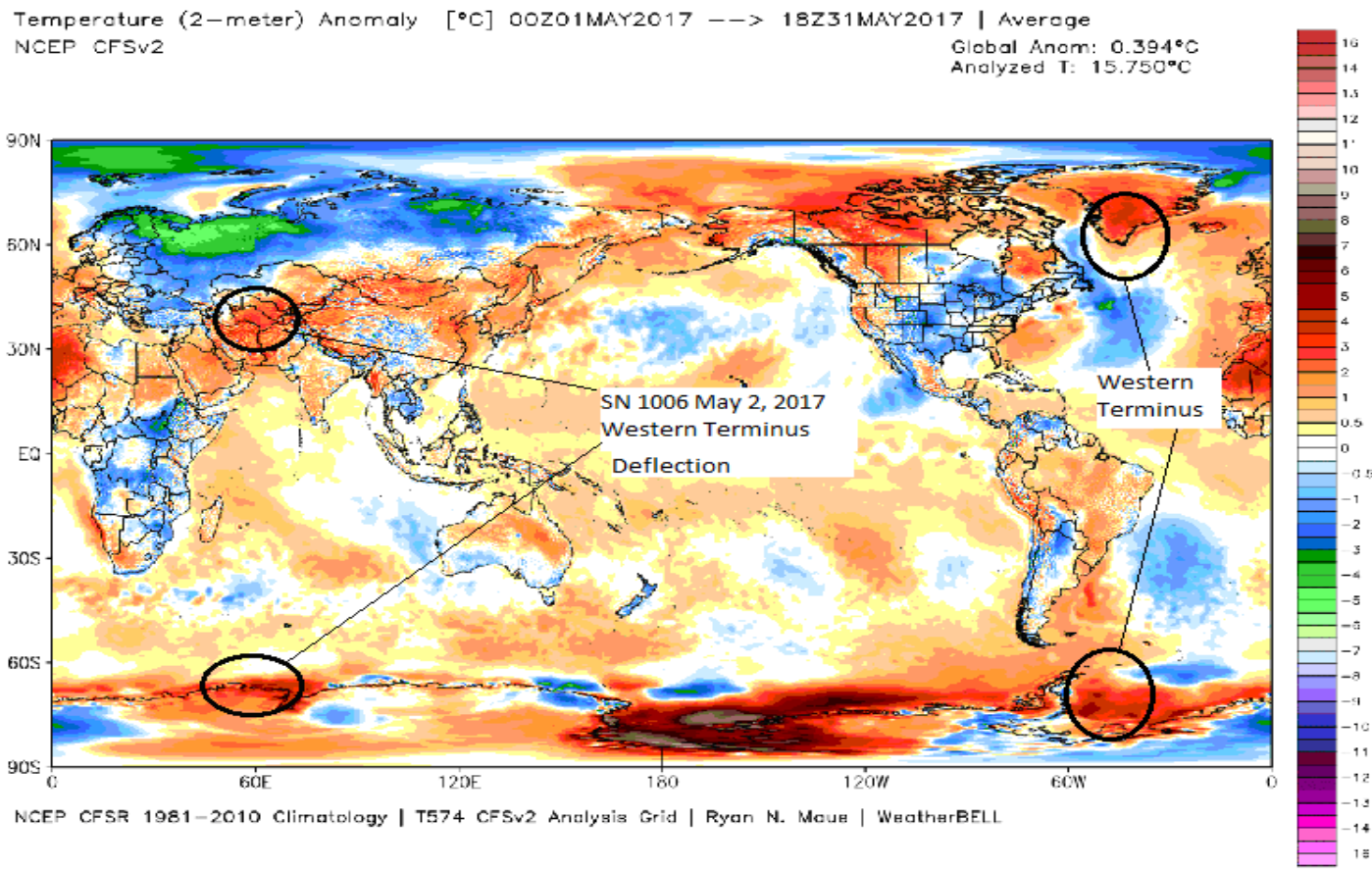

Figure 3(c). Hotspots Western Terminus and Deflection SN 1006 May 2017. 


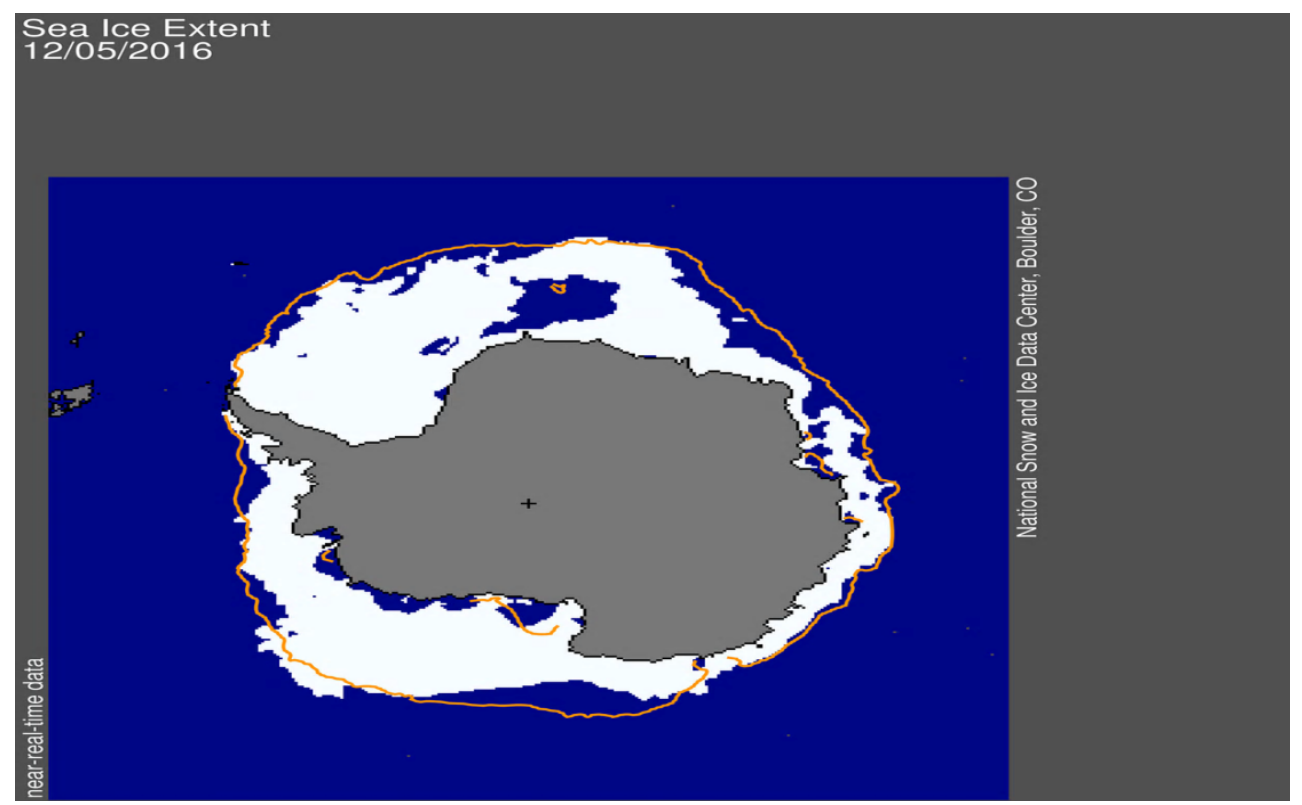

Figure 4. December 52016 New Melt Locations [16].

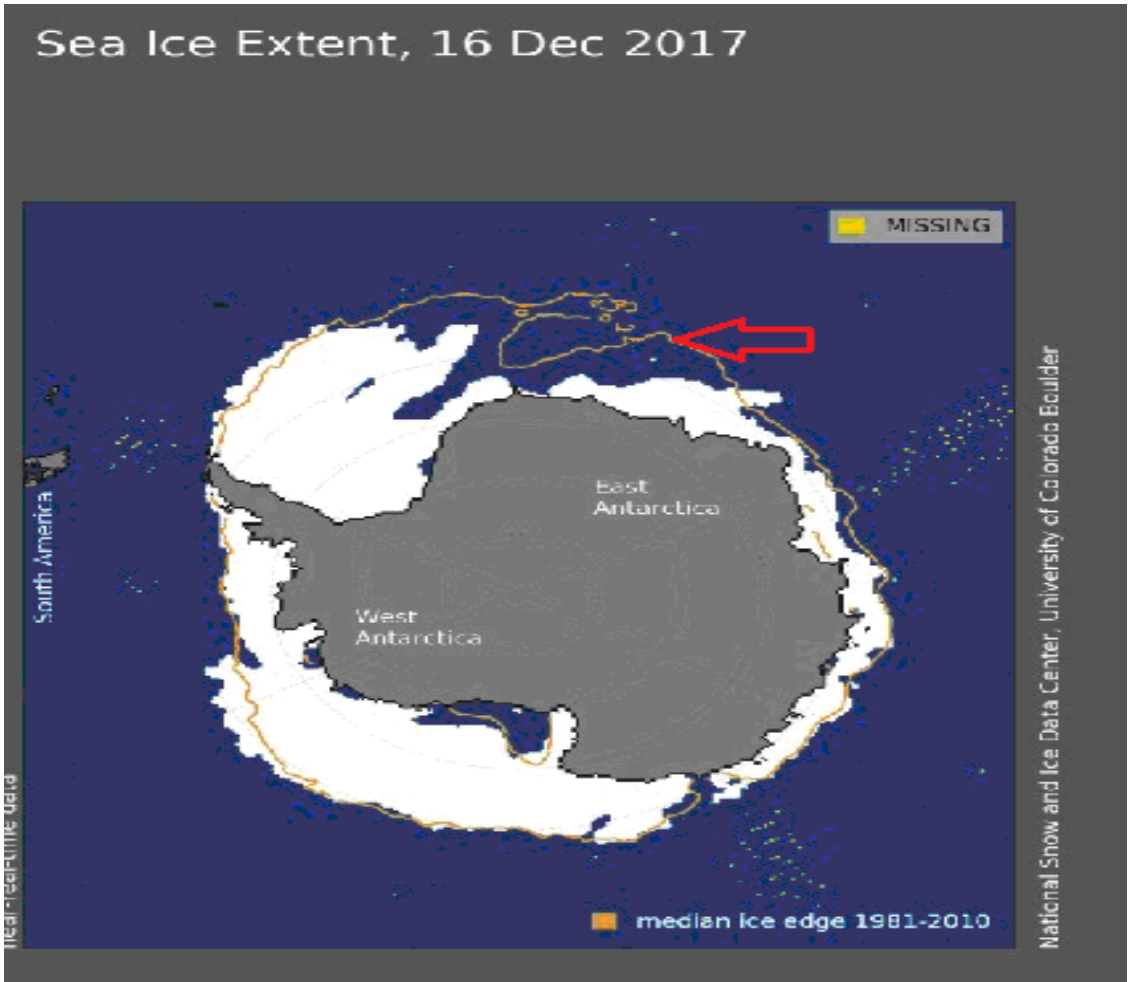

Figure 5. December 162017 New Melt Locations [16].

India in 2016 to Pakistan at 128.3 degrees Fahrenheit in 2017 [4]. The melt for the eastern terminus appears to have moved towards the continent which indicates the debris stream impact point is still moving south.

\section{Conclusion}

The time for extreme disasters due to supernovas 1006 and 1054 will be during the time of the maximum particle densities for the debris streams. For SN 11.2-0.3, this time occurred about 25 years after the ETA of 1790 . SN 1054 has been active 11 years and SN 1006 has been active 6 years. Very large problems will exist due to these SN debris streams in humanities near future.
The time of maximum density for a supernova or nova incoming debris stream will be near the center of the time period when the stream is showing ice melting effects as for the example of SN 11.2-0.3 shown in Figure 1. This defines a property of the debris stream and is consistent with Coulomb's law concerning repulsive forces of like charges. The particles in the front of the stream will increase in velocity and the particles in the end of the stream will decrease in velocity causing the radial thickness of the stream to increase. One result is the maximum density of the stream to exist at the center of the radial thickness.

It would be wise for all nations to store a year's supply of nonperishable foods for their populations as a safety factor against 
starvation in case the phenomena of a year without a Summer repeats in our future. The 180 longitudinal bands of activity for SNs 1054 and 1006 encompass nearly the entire globe as can be seen in Figure 2. As a result, any nation may be affected by a year without a summer.

The southern debris stream of SN 1006 has varying density and the sun's magnetic field is changing causing the deflected debris stream to move to the west. The western and eastern termini impact points must stay at the same longitudes, but they may move north and south to different latitudes.

Exploding star debris streams have destroyed many lives in the past and they have begun to kill many in the present. The future looks bleak unless countries band together to stop incoming particles that bring heat, cold, and disease to stop mankind from fulfilling their destiny.

Just as the flu outbreak increased in the USA due to the focal point travel for SN 1054, a similar increase of an event of SN 1006 should be expected in China and Australia beginning in December 2017 for Australia and February 2018 for China.

Epidemics caused by exploding star debris streams could be avoided by the correct application of large magnetic fields that deflect the incoming germs, but care must be applied to harvest the incoming energy from the streams to avoid making Earth a popsicle planet. Countless millions of lives could be saved by fighting the germs before they enter our biosphere and mankind could achieve its destiny.

\section{References}

1. Sokeland WP. Supernova and Nova Explosion's Space
Weather: Correlated Megafauna Extinctions and Biosphere Meg-disturbances- Global Warming. Journal of Earth Science and Engineering. 2017:136-53.

2. Sokeland WP. WZ Sagittae: Space Weather- Global Warming. Journal of Earth Science and Engineering. 2017:154-74.

3. Sokeland WP. WZ Sagittae, SN 1054, SN 1006 Space Weather- Global Warming. Journal of Earth Science and Engineering. 2017:194-216.

4. Sokeland WP. Global warming and cooling: friend and foe to mankind. 2018.

5. https://en.wikipedia.org/wiki/SN_386

6. https://en.wikipedia.org/wiki/Quadrantids

7. http://www.the-cryosphere.net/7/303/2013/tc-7-303-2013. pdf /

8. https://en.wikipedia.org/wiki/Dalton_Minimum

9. https://en.wikipedia.org/wiki/1815_eruption_of_Mount Tambora

10. https://en.wikipedia.org/wiki/Year_Without_a_Summer

11. https://wattsupwiththat.files.wordpress.com/2011/09/ weather1.pdf/

12. https://en.wikipedia.org/wiki/1811\%E2\%80\%9312_New_ Madrid_earthquakes

13. https://en.wikipedia.org/wiki/Cholera_outbreaks_and pandemics

14. https:/www.theguardian.com/environment/2016/dec/06/ arctic-antarctic-ice-melt-november-record\#img-2

\section{*Correspondence to:}

William P Sokeland

Retired Heat Transfer Expert

Spacecraft and Turbine Engines

Oakland City

Indiana 47660

USA

Tel: 812-304-9629

E-mail:wpsokeland@yahoo.com 